\title{
How Much Space Does My Shade Tree Need? Planting Space Recommendations for Medium and Large Trees in Florida Cities ${ }^{1}$
}

\author{
Deborah R. Hilbert, Andrew K. Koeser, Brooke L. Moffis, JuWanda G. Rowell, and Drew C. \\ McLean ${ }^{2}$
}

Trees provide urban landscapes with shade, beauty, and habitat. They can also help lessen the effects of flooding and urban heat buildup while storing carbon dioxide, a major greenhouse gas. When planted in the wrong place, however, trees can damage urban infrastructure like sidewalks. Most of a tree's roots, including the large structural roots, occur within the top two feet of soil. In urban settings, this means as the roots grow, they can displace or crack nearby sidewalks, roads, and curbs (Randrup et al. 2001). On the other hand, root-cutting activity that commonly occurs with the installation and repair of infrastructure can result in tree instability, decline in health, or even death (Benson et al. 2020). Shade trees such as live oaks (Quercus virginiana) are commonly planted in small planting areas, only to be removed a decade or so later when they begin damaging infrastructure or declining in health given limiting planting space.

Premature tree removal represents a significant economic and environmental loss. When determining the economic value of urban trees, one must consider the resources spent growing and maintaining the trees (e.g., nursery production costs, water, transportation, mulch, etc.), as well as the benefits they provide. When a tree is planted in the landscape, it can take years before its ecosystem services (e.g., carbon storage, shade, and stormwater mitigation) outweigh the earlier costs associated with production and planting. Once this point is reached, tree benefits continue to increase as the tree grows. By supporting the growth of healthy, long-lived trees, communities can save substantial amounts of money. For example, recent research on Florida's urban tree canopy has shown that the urban forest in the Tallahassee metropolitan area provides an estimated value of $\$ 300$ million each year by reducing air pollution, stormwater runoff, and carbon dioxide (Mclean et al. 2020). If practitioners could adjust planting practices and provide adequate space to support tree health and survival, they could increase tree benefits, including the positive effects trees have on human health and well-being (Wolf et al. 2020).

To maximize the benefits provided by urban trees, we need better-informed tree selection and larger planting spaces with the capacity to support big-canopy trees (particularly street trees). Information is available for recommended soil volume for urban trees in planting pits, which is the amount of soil in an area needed to support a tree's root system. However, minimum soil volume recommendations do not look directly at the conflict that occurs between infrastructure and the base of the tree when root space is

1. This document is ENH1328, one of a series of the Environmental Horticulture Department, UF/IFAS Extension. Original publication date October 2020. Visit the EDIS website at https://edis.ifas.ufl.edu for the currently supported version of this publication.

2. Deborah R. Hilbert, biological scientist II; Andrew K. Koeser, associate professor, Environmental Horticulture Department, UF/IFAS Gulf Coast Research and Education; Brooke L. Moffis, Extension agent III; JuWanda G. Rowell, UF/IFAS Extension Lake County; and Drew C. McLean, biological scientist II, UF/IFAS Gulf Coast REC; UF/IFAS Extension, Gainesville, FL 32611.

The Institute of Food and Agricultural Sciences (IFAS) is an Equal Opportunity Institution authorized to provide research, educational information and other services

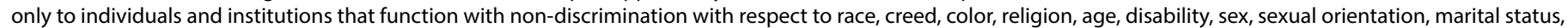

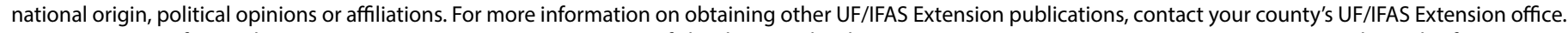
U.S. Department of Agriculture, UF/IFAS Extension Service, University of Florida, IFAS, Florida A \& M University Cooperative Extension Program, and Boards of County Commissioners Cooperating. Nick T. Place, dean for UF/IFAS Extension. 
not limited (e.g., in the space between a road curb and a sidewalk). This article provides a new piece of the puzzle by presenting recommendations on how far a tree should be planted from infrastructure using data from field measurements. This article focuses specifically at ground-level infrastructure like sidewalks and streets, not belowground utilities.

This fact sheet is intended to help arborists, urban foresters, landscape designers, landscapers, and anyone else responsible for the planting of trees in developed areas make informed decisions regarding the planting width requirements of the trees they select.

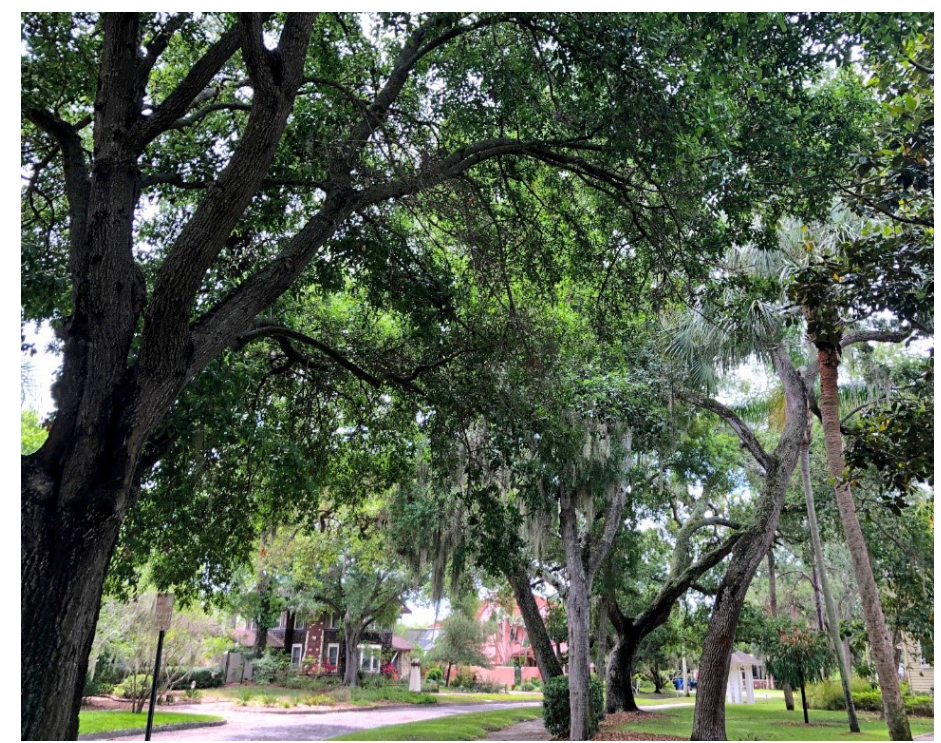

Figure 1. Residential trees like these in Pinellas County provide many benefits.

Credits: Deborah R. Hilbert, UF/IFAS

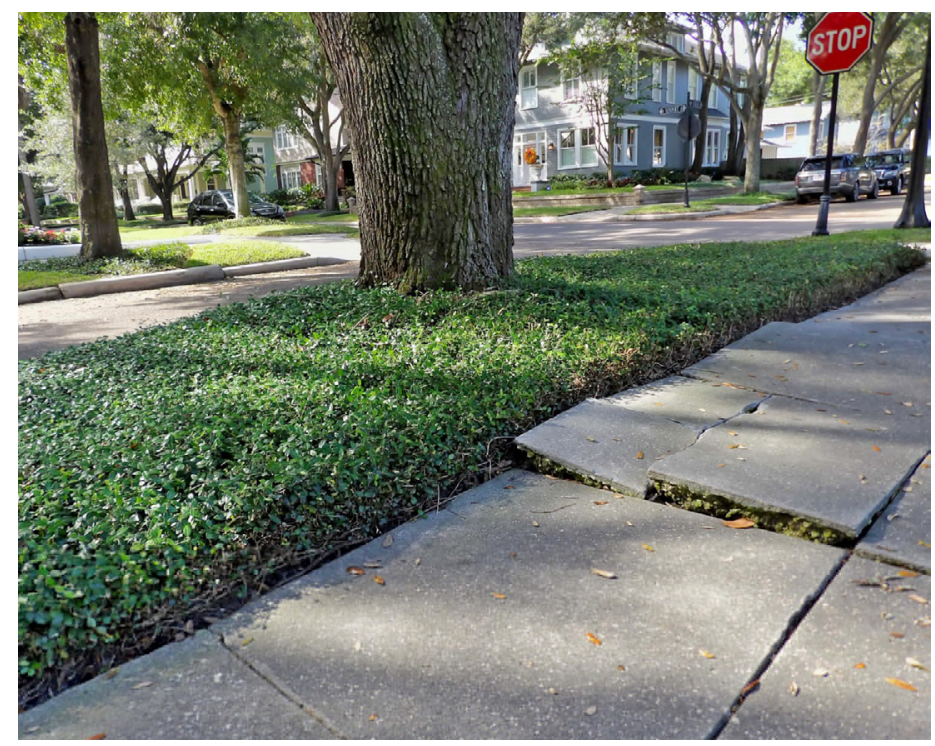

Figure 2. Sidewalk damage caused by a large southern live oak (Quercus virginiana).

Credits: Deborah R. Hilbert, UF/IFAS

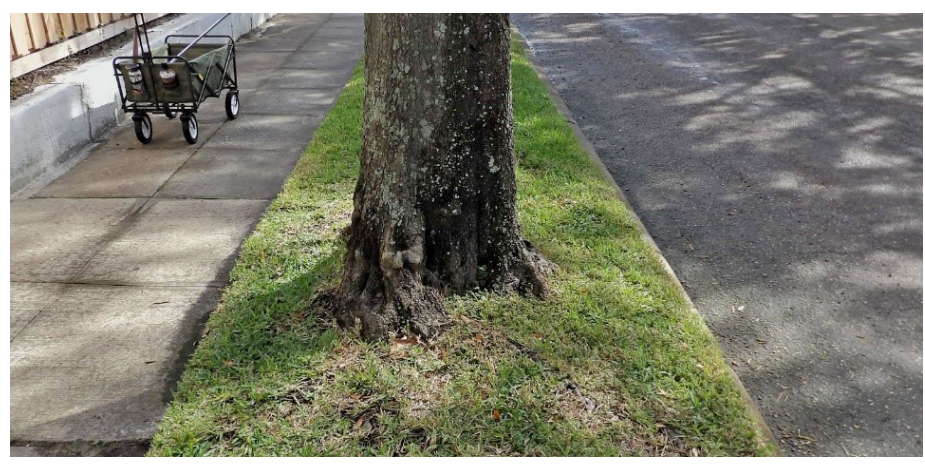

Figure 3. A large laurel oak (Quercus laurifolia) planted in a narrow planting strip. The lighter-colored pavement squares on the left were most likely replacements due to tree root damage.

Credits: Deborah R. Hilbert, UF/IFAS

\section{Applying Urban Tree Research to Planting Space Recommendations}

Prior research conducted by UF/IFAS scientists and colleagues looked at the size of common street trees at two different areas of the trunk: 4.5 feet off the ground (a common forestry measurement called diameter at breast height, or DBH) and ground level (a measurement called trunk flare diameter, or TFD) (Figure 4). It is tricky to measure the diameter of the trunk flare, so researchers created equations allowing users to predict TFD by plugging in the more common measurement, DBH (Hilbert et al. 2020; North et al. 2015). This research focused on medium- and large-stature trees (Table 1), but future work will look at small-stature trees such as crapemyrtle (Lagerstroemia indica) and Japanese privet (Ligustrum japonicum).

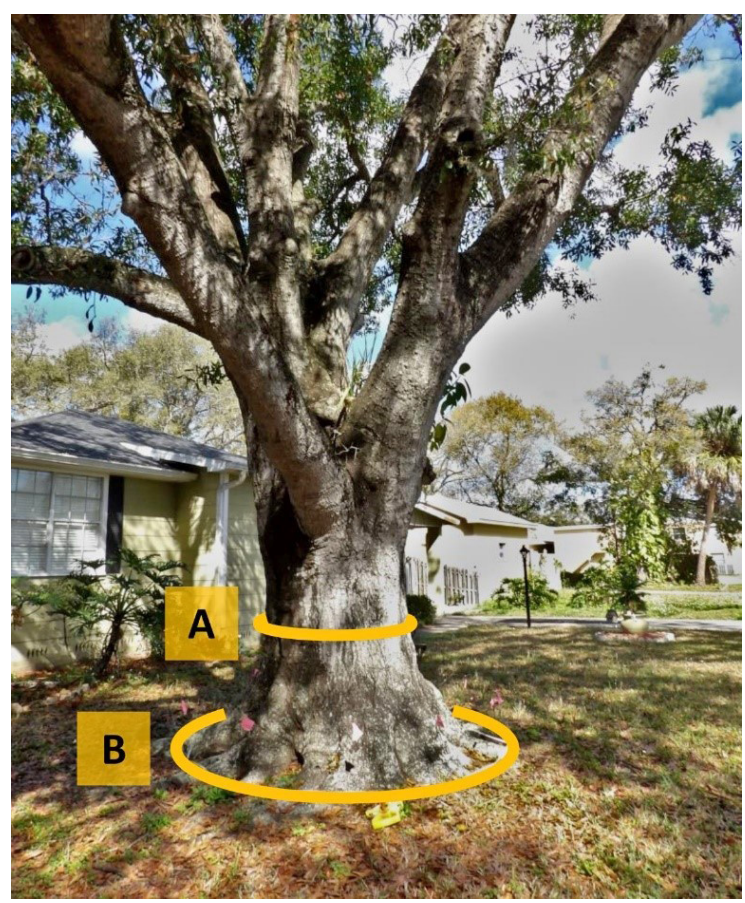

Figure 4. Researchers measured the diameters of trees at breast height (A) and at trunk flare (B) to create predictive equations. Credits: Deborah R. Hilbert, UF/IFAS 
For this article, we looked at the maximum diameter at breast height of common urban trees by searching through champion tree registers. This gave us an idea of how large a species' trunk can grow in natural conditions (i.e., the maximum genetic potential of the trees). Then we plugged those $\mathrm{DBH}$ values into a series of equations from our abovementioned research (Hilbert et al. 2020) to calculate these species' TFDs. To determine recommended planting space, we added 4 feet of buffer to either side of the trunk flare to account for large supportive roots that (while hidden below the soil surface) could still lift or crack sidewalks (Hauer et al. 2020; Johnson \& North 2016; Perry 1992). The figures below help explain this.

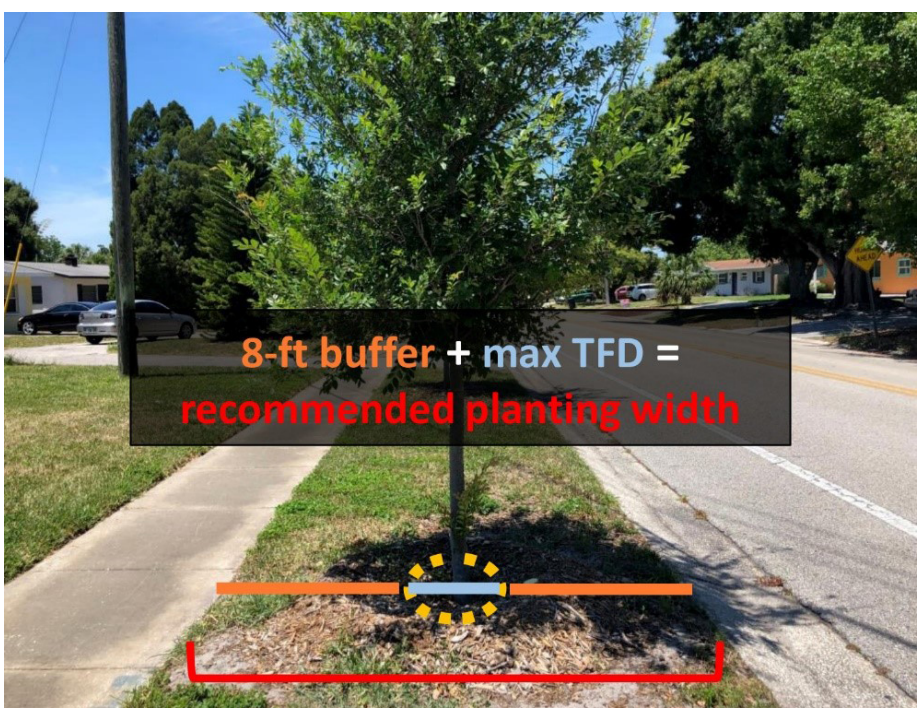

Figure 5. A Chinese elm tree (Ulmus parvifolia) in a planting strip between street and sidewalk. Recommended planting strip width is 8 feet of buffer ( 4 feet on each side) plus the expected trunk flare diameter (TFD). For this Chinese elm, that would be 10 feet total. (Figure not to scale.)

Credits: Deborah R. Hilbert, UF/IFAS

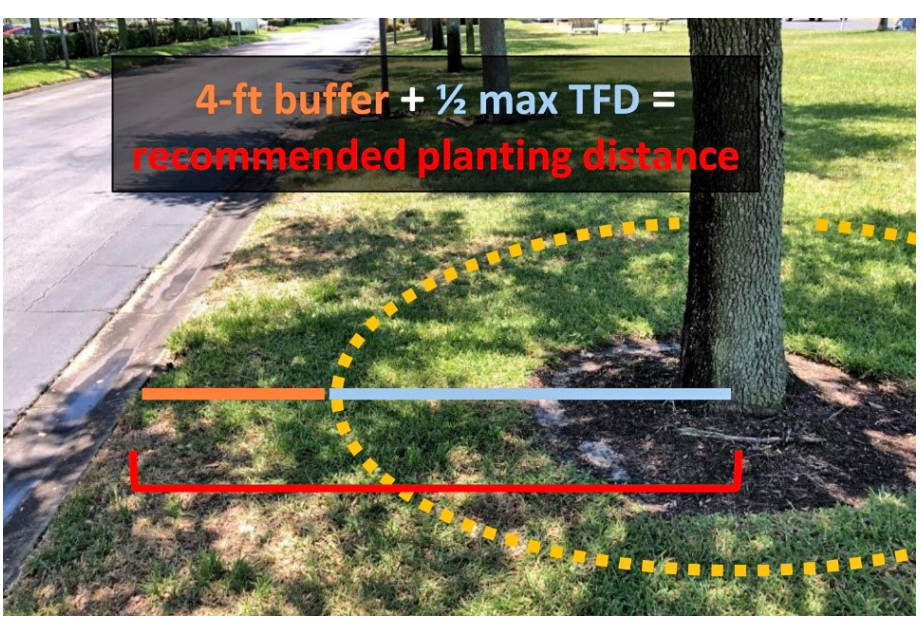

Figure 6. Live oak (Quercus virginiana) in a lawn adjacent to a street. Recommended planting distance is 4 feet of buffer plus half of the expected mature trunk flare diameter (TFD). For this live oak, that would be 13 feet total. (Figure not to scale.)

Credits: Deborah R. Hilbert, UF/IFAS

\section{How to Use the Planting Space Recommendation Tables}

The tree species in Table 1 and Table 2 are medium- and large-stature trees featured in the planting lists for $8 \mathrm{com}$ munities representing north, central, and south Florida (i.e., Fort Lauderdale, Gainesville, Miami, Minneola, Ocala, Pensacola, Tallahassee, and Tampa). Table 1 outlines species information including hardiness zones (north, central, and south Florida), status as a Florida native tree, mature tree height and spread, drought tolerance (high, moderate, and low), and the DBH from state champion tree registers.

Table 2 outlines the planting space recommendations for these species. "Planting Distance" tells you how far to plant a tree from a single road, driveway, sidewalk, etc. "Planting Width" gives the necessary width of a planting strip, such as the space between a road and sidewalk or the width of a median. See Figures 5 and 6 for help visualizing this. "Max Genetic Potential" refers to the space needed to support a tree that will grow to the diameter of the referenced champion tree. Because few trees grow to their full genetic potential, we included columns to show the planting width required to support a tree that grows to be $75 \%, 50 \%$, or $25 \%$ of the maximum size listed in Table 1 .

Table 3 can be used if the species you are interested in is not featured in Tables 1 and 2, or if you simply want to accommodate a tree to a given DBH (i.e., 10 in, 20 in, 30 in, or $40 \mathrm{in}$ ). Using this table requires some research and only applies to medium and large shade trees, not small trees. To determine the species' typical habitat, visit the USDA PLANTS database and look at its "wetland indicator" status for the Atlantic and Gulf Coastal Plain region (or whatever region your state falls within). Additionally, it may be helpful to use a champion tree register to make sure you are not selecting an expected diameter that is beyond the maximum genetic potential of the species.

Future research will need to look at how these planting space recommendations should be modified when technology like suspended sidewalks is used. We suspect the 4-foot planting buffer can be reduced if suspended sidewalks are used because large, supportive roots are situated below infrastructure in these designs. By following planting space recommendations, damage to urban infrastructure can be minimized while supporting the growth of healthy, long-lived shade trees. When planted for long-term success, mature shade trees provide ecological, financial, and environmental benefits to communities for many years. 


\section{Acknowledgments}

The authors wish to thank the following people for their contributions to the research that laid the foundation for this article: Robert J. Northrop, county Extension forester, UF/IFAS Extension Hillsborough County; Eric A. North, assistant professor, University of Nebraska-Lincoln; Richard J. Hauer, professor of forestry, University of WisconsinStevens Point; Michael Andreu, associate professor and Extension coordinator, UF/IFAS School of Forest Resources and Conservation; and Stephani Parbs, student researcher, University of Wisconsin-Stevens Point.

\section{References}

\section{Background and Equations}

Benson, A. R., A. K. Koeser, and J. Morgenroth. 2020.

"Response of mature roadside trees to root severance treatments." Urban Forestry \& Urban Greening 46 (2019): 126448

Hauer, R. J., A. K. Koeser, S. Parbs, J. Kringer, R. Krouse, K. Ottman, R. W. Miller, D. Sivyer, N. Timilsina, and L. P. Werner. 2020. "Long-term effects and development of a tree preservation program on tree condition, survival, and growth." Landscape and Urban Planning 193 (2020): 103670 .

Hilbert, D. R., E. A. North, R. J. Hauer, A. K. Koeser, D. C. McLean, R. J. Northrop, M. Andreu, and S. Parbs. 2020. "Predicting trunk flare diameter to prevent tree damage to infrastructure." Urban Forestry \& Urban Greening 49 (2020): 126645.

Johnson, G., and E. North. 2016. "Total Infrastructure Planning: Mind the four R's for successful street landscapes.” Arborist News 25 (3): 69.

McLean, D. C., A. K. Koeser, D. R. Hilbert, S. Landry, A. Abd-Elrahman, K. Britt, M. Lusk, M. Andreu, and R. Northrop. 2020. Florida's Urban Forest: A Valuation of Benefits. ENH1331. Gainesville: University of Florida Institute of Food and Agricultural Sciences. https://edis.ifas. ufl.edu/ep595

North, E. A., G. R. Johnson, and T. E. Burk. 2015. “Trunk flare diameter predictions as an infrastructure planning tool to reduce tree and sidewalk conflicts." Urban Forestry \& Urban Greening 14 (1): 65-71.
Perry, T. O. 1992. "The ecology of tree roots and the practical significance thereof." Journal of Arboriculture 8 (8): 197-211.

Randrup, T. B., E. G. McPherson, and L. R. Costello. 2001. "A review of tree root conflicts with sidewalks, curbs, and roads." Urban Ecosystems 5 (2001): 209-225.

Wolf, K. L., S. T. Lam, J. K. McKeen, G. R. A. Richardson, M. van den Bosch, and A. C. Bardekjian. 2020. "Urban trees and human health: A scoping review." Int. J. Environ. Res. Public Health 17 (12): 4371. doi:10.3390/ijerph17124371.

\section{Habitat Designation and Species Information \\ FIRST CHOICE}

FNPS. "Native plants for your area." Visited May 11, 2020. https://www.fnps.org/plants

UF/IFAS Environmental Horticulture Department. "Tree selection for urban and suburban landscapes." Visited May 11, 2020. https://hort.ifas.ufl.edu/woody/selection.shtml

USDA, NRCS. 2019. “The PLANTS Database.” National Plant Data Team, Greensboro, NC 27401-4901 USA. Visited May 11, 2020. http://plants.usda.gov

\section{SECOND CHOICE (USED IF THE ABOVE THREE DID NOT HAVE THE INFORMATION, SUCH AS FOR SOME NON-NATIVES)}

CABI. 2020. Invasive Species Compendium. Wallingford, UK: CAB International. Visited April 24, 2020. www.cabi. org/isc

Lady Bird Johnson Wildflower Center. 2020. "Native Plants of North America." Visited May 11, 2020. https://www. wildflower.org/plants-main

ISSG. 2020. "Global Invasive Species Database." Visited May 11, 2020. http://www.iucngisd.org/gisd/

IUCN. 2020. "The IUCN Red List of Threatened Species." Visited May 11, 2020. https://www.iucnredlist.org/

\section{Nomenclature}

Wunderlin, R. P., B. F. Hansen, A. R. Franck, and F. B. Essig. 2020. Atlas of Florida Plants (http://florida.plantatlas. usf.edu/). [S. M. Landry and K. N. Campbell (application development), USF Water Institute.] Tampa: Institute for Systematic Botany, University of South Florida. 


\section{Max DBH for Champion Trees from}

American Forests. 2020. “The 2020 National Register of Champion Trees." Visited May 11, 2020. https://www. americanforests.org/get-involved/americas-biggest-trees/ champion-trees-national-register/

Florida Department of Agriculture and Consumer Services. 2020. Champion Trees. Visited May 11, 2020. https://www.fdacs.gov/Forest-Wildfire/Our-Forests/ Florida-Champion-Trees

Texas A\&M Forest Service. 2020. "Big Tree Registry.” Visited May 12, 2020. http://texasforestinfo.tamu.edu/ BigTreeRegistry/Lists

Ward, D.B. and R.T. Ing. 1997. Big Trees: The Florida Register. The Florida Native Plant Society. 223 pp. 
Table 1. Species information for common medium and large native Florida trees, used to create planting space recommendations.

\begin{tabular}{|c|c|c|c|c|c|c|c|}
\hline Botanical Name & Common Name & $\begin{array}{c}\text { Zones (N, C, } \\
\mathbf{S}^{\mathrm{w}}\end{array}$ & $\begin{array}{l}\text { Mature } \\
\text { Height }\end{array}$ & $\begin{array}{l}\text { Mature } \\
\text { Spread }\end{array}$ & $\begin{array}{l}\text { Drought } \\
\text { Tolerance }^{x}\end{array}$ & $\begin{array}{l}\text { Max DBH of } \\
\text { Champion }\end{array}$ & $\begin{array}{c}\text { Computed } \\
\text { Max TFD }\end{array}$ \\
\hline Acer rubrum & red maple & $\mathrm{N}, \mathrm{C}$ & $60-75 \mathrm{ft}$ & $25-35 \mathrm{ft}$ & $\bmod$ & $57.0 \mathrm{in}$ & $7.6 \mathrm{ft}$ \\
\hline Betula nigra & river birch & $\mathrm{N}$ & $40-50 \mathrm{ft}$ & $25-35 \mathrm{ft}$ & $\bmod$ & 33.4 in & $4.7 \mathrm{ft}$ \\
\hline Bursera simaruba & gumbo limbo & $\mathrm{C}, \mathrm{S}$ & $25-50 \mathrm{ft}$ & $25-50 \mathrm{ft}$ & high & 33.1 in & $3.6 \mathrm{ft}$ \\
\hline Carpinus caroliniana & American hornbeam & $\mathrm{N}$ & $20-30 \mathrm{ft}$ & $20-30 \mathrm{ft}$ & $\bmod$ & $14.0 \mathrm{in}$ & $1.9 \mathrm{ft}$ \\
\hline Carya glabra & pignut hickory & $\mathrm{N}, \mathrm{C}$ & $50-65 \mathrm{ft}$ & $30-40 \mathrm{ft}$ & high & 44.9 in & $4.9 \mathrm{ft}$ \\
\hline Celtis laevigata & sugarberry & $\mathrm{N}, \mathrm{C}, \mathrm{S}$ & $50-70 \mathrm{ft}$ & $50-60 \mathrm{ft}$ & high & 82.5 in & $11.7 \mathrm{ft}$ \\
\hline Chrysophyllum oliviforme & devilwood & $S$ & $35-45 \mathrm{ft}$ & $18-25 \mathrm{ft}$ & high & $23.2 \mathrm{in}$ & $3.1 \mathrm{ft}$ \\
\hline Coccoloba diversifolia & pigeon plum & $\mathrm{S}$ & $20-40 \mathrm{ft}$ & $20-35 \mathrm{ft}$ & high & $26.8 \mathrm{in}$ & $2.9 \mathrm{ft}$ \\
\hline Coccoloba uvifera & seagrape & $\mathrm{C}, \mathrm{S}$ & $35-50 \mathrm{ft}$ & $20-30 \mathrm{ft}$ & high & 47.7 in & $5.2 \mathrm{ft}$ \\
\hline Ficus citrifolia & shortleaf fig & $\mathrm{S}$ & $25-50 \mathrm{ft}$ & $30-40 \mathrm{ft}$ & high & 79.0 in & $8.6 \mathrm{ft}$ \\
\hline Fraxinus pennsylvanica & green ash & $\mathrm{N}$ & $60-70 \mathrm{ft}$ & $45-50 \mathrm{ft}$ & high & 39.1 in & $4.2 \mathrm{ft}$ \\
\hline Gordonia lasianthus & loblolly bay & $\mathrm{N}, \mathrm{C}$ & $35-60 \mathrm{ft}$ & $10-15 \mathrm{ft}$ & mod & 52.2 in & $7.4 \mathrm{ft}$ \\
\hline Liquidambar styraciflua & sweetgum & $\mathrm{N}, \mathrm{C}$ & $60-75 \mathrm{ft}$ & $35-50 \mathrm{ft}$ & $\bmod$ & $51.0 \mathrm{in}$ & $6.8 \mathrm{ft}$ \\
\hline Liriodendron tulipifera & tulip poplar & $\mathrm{N}$ & $80-100 \mathrm{ft}$ & $30-50 \mathrm{ft}$ & $\bmod$ & $62.4 \mathrm{in}$ & $6.8 \mathrm{ft}$ \\
\hline Lysiloma latisiliquum & wild tamarind & $\mathrm{S}$ & $30-60 \mathrm{ft}$ & $30-50 \mathrm{ft}$ & high & 58.6 in & $6.3 \mathrm{ft}$ \\
\hline Magnolia grandiflora & southern magnolia & $\mathrm{N}, \mathrm{C}$ & $60-80 \mathrm{ft}$ & $30-40 \mathrm{ft}$ & $\bmod$ & 73.9 in & $9.9 \mathrm{ft}$ \\
\hline Magnolia virginiana & sweetbay magnolia & $\mathrm{N}, \mathrm{C}$ & $40-50 \mathrm{ft}$ & $15-25 \mathrm{ft}$ & low & 43.6 in & $6.2 \mathrm{ft}$ \\
\hline Nyssa biflora & swamp tupelo & $\mathrm{N}, \mathrm{C}$ & $35-45 \mathrm{ft}$ & $25-35 \mathrm{ft}$ & $\bmod$ & 50.3 in & $7.1 \mathrm{ft}$ \\
\hline Nyssa sylvatica & water tupelo & $\mathrm{N}, \mathrm{C}$ & $65-75 \mathrm{ft}$ & $25-35 \mathrm{ft}$ & high & 26.1 in & $3.5 \mathrm{ft}$ \\
\hline Piscidia piscipula & Jamaican dogwood & $\mathrm{C}, \mathrm{S}$ & $25-50 \mathrm{ft}$ & $25-50 \mathrm{ft}$ & high & 43.6 in & $4.7 \mathrm{ft}$ \\
\hline Platanus occidentalis & American sycamore & $\mathrm{N}$ & $75-90 \mathrm{ft}$ & $50-70 \mathrm{ft}$ & high & $75.8 \mathrm{in}$ & $10.7 \mathrm{ft}$ \\
\hline Prunus caroliniana & Carolina laurel cherry & $\mathrm{N}, \mathrm{C}$ & $25-40 \mathrm{ft}$ & $15-25 \mathrm{ft}$ & high & 19.1 in & $2.1 \mathrm{ft}$ \\
\hline Prunus serotina & black cherry & $\mathrm{N}, \mathrm{C}$ & $60-90 \mathrm{ft}$ & $35-50 \mathrm{ft}$ & high & $45.5 \mathrm{in}$ & $4.9 \mathrm{ft}$ \\
\hline Quercus falcata & southern red oak & $\mathrm{N}, \mathrm{C}$ & $60-80 \mathrm{ft}$ & $60-70 \mathrm{ft}$ & high & 102.2 in & $11.1 \mathrm{ft}$ \\
\hline Quercus geminata & sand live oak & $\mathrm{N}, \mathrm{C}, \mathrm{S}$ & $50-90 \mathrm{ft}$ & $30-50 \mathrm{ft}$ & high & 70.4 in & $7.6 \mathrm{ft}$ \\
\hline Quercus laurifolia & laurel oak & $\mathrm{N}, \mathrm{C}, \mathrm{S}$ & $60-70 \mathrm{ft}$ & $35-45 \mathrm{ft}$ & $\bmod$ & 68.8 in & $10.9 \mathrm{ft}$ \\
\hline Quercus nigra & water oak & $\mathrm{N}, \mathrm{C}, \mathrm{S}$ & $50-60 \mathrm{ft}$ & $60-70 \mathrm{ft}$ & high & 63.3 in & $8.4 \mathrm{ft}$ \\
\hline Quercus phellos & willow oak & $\mathrm{N}, \mathrm{C}$ & $60-75 \mathrm{ft}$ & $40-50 \mathrm{ft}$ & high & 18.2 in & $2.6 \mathrm{ft}$ \\
\hline Quercus shumardii & Shumard oak & $\mathrm{N}, \mathrm{C}$ & $55-80 \mathrm{ft}$ & $40-50 \mathrm{ft}$ & high & 52.2 in & $7.0 \mathrm{ft}$ \\
\hline Quercus texanaz & Texas red oak & $\mathrm{N}$ & $60-80 \mathrm{ft}$ & $35-50 \mathrm{ft}$ & $\bmod$ & $65.0 \mathrm{in}$ & $9.2 \mathrm{ft}$ \\
\hline Quercus virginiana & southern live oak & $\mathrm{N}, \mathrm{C}, \mathrm{S}$ & $60-80 \mathrm{ft}$ & $60-120 \mathrm{ft}$ & high & 130.9 in & $18.5 \mathrm{ft}$ \\
\hline Sassafras albidum & sassafras & $\mathrm{N}, \mathrm{C}$ & $30-60 \mathrm{ft}$ & $25-40 \mathrm{ft}$ & high & 30.9 in & $3.3 \mathrm{ft}$ \\
\hline Simarouba glauca & paradise-tree & $\mathrm{S}$ & $40-50 \mathrm{ft}$ & $25-30 \mathrm{ft}$ & $\bmod$ & 34.7 in & $3.8 \mathrm{ft}$ \\
\hline Swietenia mahagoni & mahogany & S & $40-60 \mathrm{ft}$ & $40-60 \mathrm{ft}$ & high & 59.6 in & $6.5 \mathrm{ft}$ \\
\hline Taxodium ascendens & pondcypress & $\mathrm{N}, \mathrm{C}$ & $50-60 \mathrm{ft}$ & $10-15 \mathrm{ft}$ & high & 80.0 in & $11.3 \mathrm{ft}$ \\
\hline Taxodium distichum & baldcypress & $\mathrm{N}, \mathrm{C}, \mathrm{S}$ & $60-80 \mathrm{ft}$ & $25-35 \mathrm{ft}$ & high & 178.3 in & $25.3 \mathrm{ft}$ \\
\hline Tilia americana & American linden & $\mathrm{N}$ & $50-80 \mathrm{ft}$ & $35-50 \mathrm{ft}$ & $\bmod$ & $25.2 \mathrm{in}$ & $2.9 \mathrm{ft}$ \\
\hline Ulmus alata & winged elm & $\mathrm{N}, \mathrm{C}$ & $45-70 \mathrm{ft}$ & $30-40 \mathrm{ft}$ & high & 40.4 in & $4.4 \mathrm{ft}$ \\
\hline Ulmus americana & American elm & $\mathrm{N}, \mathrm{C}$ & $70-90 \mathrm{ft}$ & $50-70 \mathrm{ft}$ & high & $61.1 \mathrm{in}$ & $8.1 \mathrm{ft}$ \\
\hline \multicolumn{8}{|c|}{$\begin{array}{l}{ }^{w} \mathrm{~N}, \mathrm{C} \text { and S stand for north, central, and south Florida. } \\
\mathrm{x} \text { "mod" stands for moderate. } \\
\text { y"Max DBH of Champion" refers to the maximum diameter at breast height found in the state and big tree registers. This is the number that } \\
\text { was plugged into equations to create the planting space recommendations. }\end{array}$} \\
\hline
\end{tabular}


Table 2. Planting space recommendations based on big-tree records and equations from UF/IFAS researchers. The final three sections provide the planting space recommendations for trees if they were to reach $75 \%, 50 \%$, or $25 \%$ of the champion tree diameter.

\begin{tabular}{|c|c|c|c|c|c|c|c|c|c|}
\hline \multirow[t]{2}{*}{ Botanical Name } & \multirow[t]{2}{*}{ Common Name } & \multicolumn{2}{|c|}{$\begin{array}{c}\text { Max Genetic } \\
\text { Potential }^{\mathrm{w}}\end{array}$} & \multicolumn{2}{|c|}{$\begin{array}{c}\text { 75\% of Max Genetic } \\
\text { Potential }\end{array}$} & \multicolumn{2}{|c|}{$\begin{array}{c}50 \% \text { of Max Genetic } \\
\text { Potential }\end{array}$} & \multicolumn{2}{|c|}{$\begin{array}{c}25 \% \text { of Max Genetic } \\
\text { Potential }\end{array}$} \\
\hline & & $\begin{array}{l}\text { Planting } \\
\text { Distance }^{\mathrm{x}}\end{array}$ & $\begin{array}{l}\text { Planting } \\
\text { Width }^{y}\end{array}$ & $\begin{array}{l}\text { Planting } \\
\text { Distance }\end{array}$ & $\begin{array}{l}\text { Planting } \\
\text { Width }\end{array}$ & $\begin{array}{l}\text { Planting } \\
\text { Distance }\end{array}$ & $\begin{array}{l}\text { Planting } \\
\text { Width }\end{array}$ & $\begin{array}{l}\text { Planting } \\
\text { Distance }\end{array}$ & $\begin{array}{l}\text { Planting } \\
\text { Width }\end{array}$ \\
\hline Acer rubrum & red maple & $8 \mathrm{ft}$ & $16 \mathrm{ft}$ & $7 \mathrm{ft}$ & $14 \mathrm{ft}$ & $6 \mathrm{ft}$ & $12 \mathrm{ft}$ & $5 \mathrm{ft}$ & $10 \mathrm{ft}$ \\
\hline Betula nigra & river birch & $6 \mathrm{ft}$ & $13 \mathrm{ft}$ & $6 \mathrm{ft}$ & $12 \mathrm{ft}$ & $5 \mathrm{ft}$ & $10 \mathrm{ft}$ & $5 \mathrm{ft}$ & $9 \mathrm{ft}$ \\
\hline Bursera simaruba & gumbo limbo & $6 \mathrm{ft}$ & $12 \mathrm{ft}$ & $5 \mathrm{ft}$ & $11 \mathrm{ft}$ & $5 \mathrm{ft}$ & $10 \mathrm{ft}$ & $4 \mathrm{ft}$ & $9 \mathrm{ft}$ \\
\hline Carpinus caroliniana & American hornbeam & $5 \mathrm{ft}$ & $10 \mathrm{ft}$ & $5 \mathrm{ft}$ & $9 \mathrm{ft}$ & $4 \mathrm{ft}$ & $9 \mathrm{ft}$ & $4 \mathrm{ft}$ & $8 \mathrm{ft}$ \\
\hline Carya glabra & pignut hickory & $6 \mathrm{ft}$ & $13 \mathrm{ft}$ & $6 \mathrm{ft}$ & $12 \mathrm{ft}$ & $5 \mathrm{ft}$ & $10 \mathrm{ft}$ & $5 \mathrm{ft}$ & $9 \mathrm{ft}$ \\
\hline Celtis laevigata & sugarberry & $10 \mathrm{ft}$ & $20 \mathrm{ft}$ & $8 \mathrm{ft}$ & $17 \mathrm{ft}$ & $7 \mathrm{ft}$ & $14 \mathrm{ft}$ & $5 \mathrm{ft}$ & $11 \mathrm{ft}$ \\
\hline $\begin{array}{l}\text { Chrysophyllum } \\
\text { oliviforme }\end{array}$ & devilwood & $6 \mathrm{ft}$ & $11 \mathrm{ft}$ & $5 \mathrm{ft}$ & $10 \mathrm{ft}$ & $5 \mathrm{ft}$ & $10 \mathrm{ft}$ & $4 \mathrm{ft}$ & $9 \mathrm{ft}$ \\
\hline Coccoloba diversifolia & pigeon plum & $5 \mathrm{ft}$ & $11 \mathrm{ft}$ & $5 \mathrm{ft}$ & $10 \mathrm{ft}$ & $5 \mathrm{ft}$ & $9 \mathrm{ft}$ & $4 \mathrm{ft}$ & $9 \mathrm{ft}$ \\
\hline Coccoloba uvifera & seagrape & $7 \mathrm{ft}$ & $13 \mathrm{ft}$ & $6 \mathrm{ft}$ & $12 \mathrm{ft}$ & $5 \mathrm{ft}$ & $11 \mathrm{ft}$ & $5 \mathrm{ft}$ & $9 \mathrm{ft}$ \\
\hline Ficus citrifolia & shortleaf fig & $8 \mathrm{ft}$ & $17 \mathrm{ft}$ & $7 \mathrm{ft}$ & $14 \mathrm{ft}$ & $6 \mathrm{ft}$ & $12 \mathrm{ft}$ & $5 \mathrm{ft}$ & $10 \mathrm{ft}$ \\
\hline $\begin{array}{l}\text { Fraxinus } \\
\text { pennsy/vanica }\end{array}$ & green ash & $6 \mathrm{ft}$ & $12 \mathrm{ft}$ & $6 \mathrm{ft}$ & $11 \mathrm{ft}$ & $5 \mathrm{ft}$ & $10 \mathrm{ft}$ & $5 \mathrm{ft}$ & $9 \mathrm{ft}$ \\
\hline Gordonia lasianthus & loblolly bay & $8 \mathrm{ft}$ & $15 \mathrm{ft}$ & $7 \mathrm{ft}$ & $14 \mathrm{ft}$ & $6 \mathrm{ft}$ & $12 \mathrm{ft}$ & $5 \mathrm{ft}$ & $10 \mathrm{ft}$ \\
\hline $\begin{array}{l}\text { Liquidambar } \\
\text { styraciflua }\end{array}$ & sweetgum & $7 \mathrm{ft}$ & $15 \mathrm{ft}$ & $7 \mathrm{ft}$ & $13 \mathrm{ft}$ & $6 \mathrm{ft}$ & $11 \mathrm{ft}$ & $5 \mathrm{ft}$ & $10 \mathrm{ft}$ \\
\hline Liriodendron tulipifera & tulip poplar & $7 \mathrm{ft}$ & $15 \mathrm{ft}$ & $7 \mathrm{ft}$ & $13 \mathrm{ft}$ & $6 \mathrm{ft}$ & $11 \mathrm{ft}$ & $5 \mathrm{ft}$ & $10 \mathrm{ft}$ \\
\hline Lysiloma latisiliquum & wild tamarind & $7 \mathrm{ft}$ & $14 \mathrm{ft}$ & $6 \mathrm{ft}$ & $13 \mathrm{ft}$ & $6 \mathrm{ft}$ & $11 \mathrm{ft}$ & $5 \mathrm{ft}$ & $10 \mathrm{ft}$ \\
\hline Magnolia grandiflora & southern magnolia & $9 \mathrm{ft}$ & $18 \mathrm{ft}$ & $8 \mathrm{ft}$ & $15 \mathrm{ft}$ & $6 \mathrm{ft}$ & $13 \mathrm{ft}$ & $5 \mathrm{ft}$ & $10 \mathrm{ft}$ \\
\hline Magnolia virginiana & sweetbay magnolia & $7 \mathrm{ft}$ & $14 \mathrm{ft}$ & $6 \mathrm{ft}$ & $13 \mathrm{ft}$ & $6 \mathrm{ft}$ & $11 \mathrm{ft}$ & $5 \mathrm{ft}$ & $10 \mathrm{ft}$ \\
\hline Nyssa biflora & swamp tupelo & $8 \mathrm{ft}$ & $15 \mathrm{ft}$ & $7 \mathrm{ft}$ & $13 \mathrm{ft}$ & $6 \mathrm{ft}$ & $12 \mathrm{ft}$ & $5 \mathrm{ft}$ & $10 \mathrm{ft}$ \\
\hline Nyssa sylvatica & water tupelo & $6 \mathrm{ft}$ & $11 \mathrm{ft}$ & $5 \mathrm{ft}$ & $11 \mathrm{ft}$ & $5 \mathrm{ft}$ & $10 \mathrm{ft}$ & $4 \mathrm{ft}$ & $9 \mathrm{ft}$ \\
\hline Piscidia piscipula & Jamaican dogwood & $6 \mathrm{ft}$ & $13 \mathrm{ft}$ & $6 \mathrm{ft}$ & $12 \mathrm{ft}$ & $5 \mathrm{ft}$ & $10 \mathrm{ft}$ & $5 \mathrm{ft}$ & $9 \mathrm{ft}$ \\
\hline Platanus occidentalis & American sycamore & $9 \mathrm{ft}$ & $19 \mathrm{ft}$ & $8 \mathrm{ft}$ & $16 \mathrm{ft}$ & $7 \mathrm{ft}$ & $13 \mathrm{ft}$ & $5 \mathrm{ft}$ & $11 \mathrm{ft}$ \\
\hline Prunus caroliniana & Carolina laurel cherry & $5 \mathrm{ft}$ & $10 \mathrm{ft}$ & $5 \mathrm{ft}$ & $10 \mathrm{ft}$ & $5 \mathrm{ft}$ & $9 \mathrm{ft}$ & $4 \mathrm{ft}$ & $9 \mathrm{ft}$ \\
\hline Prunus serotina & black cherry & $6 \mathrm{ft}$ & $13 \mathrm{ft}$ & $6 \mathrm{ft}$ & $12 \mathrm{ft}$ & $5 \mathrm{ft}$ & $10 \mathrm{ft}$ & $5 \mathrm{ft}$ & $9 \mathrm{ft}$ \\
\hline Quercus falcata & southern red oak & $10 \mathrm{ft}$ & $19 \mathrm{ft}$ & $8 \mathrm{ft}$ & $16 \mathrm{ft}$ & $7 \mathrm{ft}$ & $14 \mathrm{ft}$ & $5 \mathrm{ft}$ & $11 \mathrm{ft}$ \\
\hline Quercus geminata & sand live oak & $8 \mathrm{ft}$ & $16 \mathrm{ft}$ & $7 \mathrm{ft}$ & $14 \mathrm{ft}$ & $6 \mathrm{ft}$ & $12 \mathrm{ft}$ & $5 \mathrm{ft}$ & $10 \mathrm{ft}$ \\
\hline Quercus laurifolia & laurel oak & $9 \mathrm{ft}$ & $19 \mathrm{ft}$ & $8 \mathrm{ft}$ & $16 \mathrm{ft}$ & $7 \mathrm{ft}$ & $13 \mathrm{ft}$ & $5 \mathrm{ft}$ & $11 \mathrm{ft}$ \\
\hline Quercus nigra & water oak & $8 \mathrm{ft}$ & $16 \mathrm{ft}$ & $7 \mathrm{ft}$ & $14 \mathrm{ft}$ & $6 \mathrm{ft}$ & $12 \mathrm{ft}$ & $5 \mathrm{ft}$ & $10 \mathrm{ft}$ \\
\hline Quercus phellos & willow oak & $5 \mathrm{ft}$ & $11 \mathrm{ft}$ & $5 \mathrm{ft}$ & $10 \mathrm{ft}$ & $5 \mathrm{ft}$ & $9 \mathrm{ft}$ & $4 \mathrm{ft}$ & $9 \mathrm{ft}$ \\
\hline Quercus shumardii & Shumard oak & $7 \mathrm{ft}$ & $15 \mathrm{ft}$ & $7 \mathrm{ft}$ & $13 \mathrm{ft}$ & $6 \mathrm{ft}$ & $11 \mathrm{ft}$ & $5 \mathrm{ft}$ & $10 \mathrm{ft}$ \\
\hline Quercus texana & Texas red oak & $9 \mathrm{ft}$ & $17 \mathrm{ft}$ & $7 \mathrm{ft}$ & $15 \mathrm{ft}$ & $6 \mathrm{ft}$ & $13 \mathrm{ft}$ & $5 \mathrm{ft}$ & $10 \mathrm{ft}$ \\
\hline Quercus virginiana & southern live oak & $13 \mathrm{ft}$ & $27 \mathrm{ft}$ & $11 \mathrm{ft}$ & $22 \mathrm{ft}$ & $9 \mathrm{ft}$ & $17 \mathrm{ft}$ & $6 \mathrm{ft}$ & $13 \mathrm{ft}$ \\
\hline Sassafras albidum & sassafras & $6 \mathrm{ft}$ & $11 \mathrm{ft}$ & $5 \mathrm{ft}$ & $11 \mathrm{ft}$ & $5 \mathrm{ft}$ & $10 \mathrm{ft}$ & $4 \mathrm{ft}$ & $9 \mathrm{ft}$ \\
\hline Simarouba glauca & paradise-tree & $6 \mathrm{ft}$ & $12 \mathrm{ft}$ & $5 \mathrm{ft}$ & $11 \mathrm{ft}$ & $5 \mathrm{ft}$ & $10 \mathrm{ft}$ & $4 \mathrm{ft}$ & $9 \mathrm{ft}$ \\
\hline Swietenia mahagoni & mahogany & $7 \mathrm{ft}$ & $14 \mathrm{ft}$ & $6 \mathrm{ft}$ & $13 \mathrm{ft}$ & $6 \mathrm{ft}$ & $11 \mathrm{ft}$ & $5 \mathrm{ft}$ & $10 \mathrm{ft}$ \\
\hline Taxodium ascendens & pondcypress & $10 \mathrm{ft}$ & $19 \mathrm{ft}$ & $8 \mathrm{ft}$ & $17 \mathrm{ft}$ & $7 \mathrm{ft}$ & $14 \mathrm{ft}$ & $5 \mathrm{ft}$ & $11 \mathrm{ft}$ \\
\hline Taxodium distichum & baldcypress & $17 \mathrm{ft}$ & $33 \mathrm{ft}$ & $13 \mathrm{ft}$ & $27 \mathrm{ft}$ & $10 \mathrm{ft}$ & $21 \mathrm{ft}$ & $7 \mathrm{ft}$ & $14 \mathrm{ft}$ \\
\hline Tilia americana & American linden & $5 \mathrm{ft}$ & $11 \mathrm{ft}$ & $5 \mathrm{ft}$ & $10 \mathrm{ft}$ & $5 \mathrm{ft}$ & $9 \mathrm{ft}$ & $4 \mathrm{ft}$ & $9 \mathrm{ft}$ \\
\hline Ulmus alata & winged elm & $6 \mathrm{ft}$ & $12 \mathrm{ft}$ & $6 \mathrm{ft}$ & $11 \mathrm{ft}$ & $5 \mathrm{ft}$ & $10 \mathrm{ft}$ & $5 \mathrm{ft}$ & $9 \mathrm{ft}$ \\
\hline
\end{tabular}




\begin{tabular}{|c|c|c|c|c|c|c|c|c|c|}
\hline \multirow[t]{2}{*}{ Botanical Name } & \multirow[t]{2}{*}{ Common Name } & \multicolumn{2}{|c|}{$\begin{array}{l}\text { Max Genetic } \\
\text { Potential }^{\mathrm{w}}\end{array}$} & \multicolumn{2}{|c|}{$\begin{array}{c}\text { 75\% of Max Genetic } \\
\text { Potential }\end{array}$} & \multicolumn{2}{|c|}{$\begin{array}{c}50 \% \text { of Max Genetic } \\
\text { Potential }\end{array}$} & \multicolumn{2}{|c|}{$\begin{array}{c}25 \% \text { of Max Genetic } \\
\text { Potential }\end{array}$} \\
\hline & & $\begin{array}{l}\text { Planting } \\
\text { Distance }^{x}\end{array}$ & $\begin{array}{l}\text { Planting } \\
\text { Width }^{y}\end{array}$ & $\begin{array}{l}\text { Planting } \\
\text { Distance }\end{array}$ & $\begin{array}{l}\text { Planting } \\
\text { Width }\end{array}$ & $\begin{array}{l}\text { Planting } \\
\text { Distance }\end{array}$ & $\begin{array}{l}\text { Planting } \\
\text { Width }\end{array}$ & $\begin{array}{l}\text { Planting } \\
\text { Distance }\end{array}$ & $\begin{array}{l}\text { Planting } \\
\text { Width }\end{array}$ \\
\hline Ulmus americana & American elm & $8 \mathrm{ft}$ & $16 \mathrm{ft}$ & $7 \mathrm{ft}$ & $14 \mathrm{ft}$ & $6 \mathrm{ft}$ & $12 \mathrm{ft}$ & $5 \mathrm{ft}$ & $10 \mathrm{ft}$ \\
\hline
\end{tabular}

w "Max Genetic Potential" refers to the maximum diameter at breast height found in Florida's big tree register. It is challenging to predict how large a tree will grow in urban settings. To avoid conflict and future costs, we suggest giving the tree the maximum recommended planting space.

“"Planting Distance" tells you how far to plant a tree from a single road, driveway, sidewalk, or other ground-level infrastructure.

y "Planting Width" gives the necessary width of a planting strip such as the space between a road and sidewalk or the width of a median.

${ }^{z}$ Diameter for Quercus texana was obtained from the Texas Big Tree Registry because Florida data was not available.

Table 3. Planting space recommendations based on habitat categories and example diameters. These give rough estimates of planting space recommendations for medium and large trees that are not featured in Tables 1 and 2.

\begin{tabular}{|c|c|c|c|c|c|c|c|c|}
\hline \multirow[b]{2}{*}{$\begin{array}{l}\text { Typical } \\
\text { Habitat }^{w}\end{array}$} & \multicolumn{2}{|c|}{ 10-inch Expected DBH ${ }^{x}$} & \multicolumn{2}{|c|}{ 20-inch Expected DBH } & \multicolumn{2}{|c|}{ 30-inch Expected DBH } & \multicolumn{2}{|c|}{ 40-inch Expected DBH } \\
\hline & $\begin{array}{l}\text { Planting } \\
\text { Distance }^{y}\end{array}$ & $\begin{array}{c}\text { Planting } \\
\text { Strip Width }\end{array}$ & $\begin{array}{l}\text { Planting } \\
\text { Distance }\end{array}$ & $\begin{array}{c}\text { Planting } \\
\text { Strip Width }\end{array}$ & $\begin{array}{l}\text { Planting } \\
\text { Distance }\end{array}$ & $\begin{array}{c}\text { Planting } \\
\text { Strip Width }\end{array}$ & $\begin{array}{l}\text { Planting } \\
\text { Distance }\end{array}$ & $\begin{array}{c}\text { Planting } \\
\text { Strip Width }\end{array}$ \\
\hline Upland & $5 \mathrm{ft}$ & $9 \mathrm{ft}$ & $5 \mathrm{ft}$ & $10 \mathrm{ft}$ & $6 \mathrm{ft}$ & $11 \mathrm{ft}$ & $6 \mathrm{ft}$ & $12 \mathrm{ft}$ \\
\hline Variable & $5 \mathrm{ft}$ & $9 \mathrm{ft}$ & $5 \mathrm{ft}$ & $11 \mathrm{ft}$ & $6 \mathrm{ft}$ & $12 \mathrm{ft}$ & $7 \mathrm{ft}$ & $13 \mathrm{ft}$ \\
\hline Wetland & $5 \mathrm{ft}$ & $9 \mathrm{ft}$ & $5 \mathrm{ft}$ & $11 \mathrm{ft}$ & $6 \mathrm{ft}$ & $12 \mathrm{ft}$ & $7 \mathrm{ft}$ & $14 \mathrm{ft}$ \\
\hline
\end{tabular}

